\title{
Weighted relationship theorems and Ramanujan expansions
}

\author{
by
}

\author{
LuTz LuCHT (Clausthal)
}

1. Introduction. In 1918 Ramanujan [12] investigated the sums $c_{n}(a)$ of the $a$ th powers of all $n$th primitive roots of unity and proved that numerous arithmetical functions $g: \mathbb{N} \rightarrow \mathbb{C}$ possess an expansion of the form

$$
g(a)=\sum_{n=1}^{\infty} \widehat{g}(n) c_{n}(a)
$$

with suitable coefficients $\widehat{g}(n)$ for $n \in \mathbb{N}$. Via Dirichlet series, Hardy [6] shortly afterwards found a different approach to these Ramanujan expansions, and in 1932 Carmichael [1] gave generalizations based on certain orthogonality relations for the Ramanujan sums $c_{n}(a)$. Later on, the existence of many expansions of the type (1) could be explained by putting them into the context of harmonic analysis by which the consideration was restricted to almost even arithmetical functions $g$ having, at least in general, a nonzero asymptotic mean value (see, for example, Wintner [22], Cohen [2], Schwarz and Spilker [18], Knopfmacher [9]). The progress achieved in mean value theorems for multiplicative functions by Delange [3], Halász [5], Elliott [4] led to certain classes of multiplicative functions $g$ having a pointwise convergent Ramanujan expansion (1) (compare Schwarz [14]-[17], Tuttas [20], Warlimont [21]). From this point of view, for example the expansions (see Ramanujan [12])

$$
\begin{gathered}
d(a)=-\sum_{n=1}^{\infty} \frac{\log n}{n} c_{n}(a), \\
r(a)=\pi \sum_{n=1}^{\infty} \frac{(-1)^{n-1}}{2 n-1} c_{2 n-1}(a)
\end{gathered}
$$

of the divisor function $d(a)$ or the function $r(a)$, which counts the number of representations of $a \in \mathbb{N}$ as sum of two squares of integers, remain mysterious, and Knopfmacher [9] asks for a deeper conceptual explanation. 
The aim of this note is to develop such a concept for multiplicative arithmetical functions from the close relationship between all Ramanujan sums $c_{n}(a)$ and the good knowledge of the behaviour of the zeta function and its derivatives in the half plane $\operatorname{Re} s \geq 1$.

We shall start with weighted convolution algebras over countable commutative semigroups with unit element, and obtain a weighted inversion theorem (Theorem 1) for power series of the Wiener type. This serves as main tool in the investigation of the structure of certain classes of multiplicative functions on arithmetical semigroups with respect to the Dirichlet convolution (Theorem 2). As a consequence we obtain a general weighted relationship theorem (Theorem 3) for multiplicative functions, which leads to a summation theorem (Theorem 4) for convergent Ramanujan series and to the characterization (Theorem 5) of extensive classes of multiplicative functions having a pointwise convergent representation of the type (1). In this framework formulae (2) and (3) turn out to be very specific cases of general statements.

2. Weighted convolution algebras. Let $(H, \circ)$ be a countable commutative semigroup with unit element $e$. Denote by $W(H, \circ)$ the set of weight functions $\omega: H \rightarrow \mathbb{R}_{+}$having the properties

$$
\begin{gathered}
\omega(e)=1 \leq \omega(m \circ n) \leq \omega(m) \omega(n) \quad \text { for all } m, n \in H, \\
\inf \left\{\sqrt[\nu]{\omega\left(n^{\nu}\right)} \mid \nu \in \mathbb{N}\right\}=1 \quad \text { for all } n \in H
\end{gathered}
$$

(here we write $n^{\nu}=n \circ \ldots \circ n$ with $\nu$ "factors" $\left.n \in H\right)$. For $\omega \in W(H, \circ)$ put further $\mathcal{A}_{\omega}(H, \circ)=\left\{a: H \rightarrow \mathbb{C} \mid\|a\|_{\omega}<\infty\right\}$, where the norm is given by

$$
\|a\|_{\omega}=\sum_{n \in H}|a(n)| \omega(n),
$$

define $\lambda a+\mu b$ for $\lambda, \mu \in \mathbb{C}, a, b \in \mathcal{A}_{\omega}(H, \circ)$ pointwise, and introduce the convolution product $a * b \in \mathcal{A}_{\omega}(H, \circ)$ by

$$
(a * b)(n)=\sum_{\substack{d, m \in H \\ d \circ m=n}} a(d) b(m) \quad(n \in H) .
$$

Then, by (4), $\mathcal{A}_{\omega}(H, \circ)$ is a commutative Banach algebra with unit element $\varepsilon$,

$$
\varepsilon(n)=\left\{\begin{array}{ll}
1 & \text { if } n=e \\
0 & \text { otherwise }
\end{array} \quad(n \in H) .\right.
$$

Specifically, choose for $H$ the additive semigroup $\mathbb{N}_{0}=\mathbb{N} \cup\{0\}$, denote by $U$ the closed unit disk in the complex plane, and define for $a \in \mathcal{A}_{\omega}=$ $\mathcal{A}_{\omega}\left(\mathbb{N}_{0},+\right)$, 
Obviously the subset

$$
t_{a}(z)=\sum_{n=0}^{\infty} a(n) z^{n} \quad(z \in U) .
$$

$$
\mathcal{A}_{\omega}^{*}=\left\{a \in \mathcal{A}_{\omega} \mid t_{a}(z) \neq 0 \text { for all } z \in U\right\} \subset \mathcal{A}_{\omega}
$$

is closed under the convolution $*$ which corresponds to the Cauchy product of power series, and $\varepsilon \in \mathcal{A}_{\omega}^{*}$ since $t_{\varepsilon}(z)=1$. Wiener's inversion theorem for power series (see, for example, Rudin [13], Theorem 18.21) now takes the following weighted form.

TheOREm 1. For $\omega \in W\left(\mathbb{N}_{0},+\right), \mathcal{A}_{\omega}^{*}$ is the group of invertible elements in $\mathcal{A}_{\omega}$ with respect to the convolution $*$.

For the proof consider any multiplicative linear functional $h$, element of the maximal ideal space $\Delta$ of $\mathcal{A}_{\omega}$. Then $h(a)=t_{a}(\zeta)$ for some $\zeta \in \mathbb{C}$ satisfying $|\zeta|^{n} \leq \omega(n)$ for all $n \in \mathbb{N}$, and (5) implies $\zeta \in U$. Notice that $a \in \mathcal{A}_{\omega}$ is invertible if and only if $h(a) \neq 0$ for every $h \in \Delta$ (see again Rudin [13], Theorem 18.17) which is equivalent to $a \in \mathcal{A}_{\omega}^{*}$.

3. Multiplicative functions on arithmetical semigroups. The concept of arithmetical semigroups $(H, \circ)$ is based on the model $(\mathbb{N}, \cdot)$. By definition there is a non-empty set $P \subset H$ of free generators, the prime elements, such that the Unique Factorization Theorem holds in $H$, and there is a completely multiplicative norm mapping $|\cdot|: H \rightarrow \mathbb{R}_{+}$such that the number of elements with every finite bound is finite. This implies, for example, that $H$ is countable. The usual notions of elementary number theory, like divisor, Dirichlet convolution, multiplicative function, are definable in arithmetical semigroups (see Knopfmacher [9]). Specifically, denote by $\mathcal{M}$ the set of multiplicative functions $f: H \rightarrow \mathbb{C}$, i.e. $f(e)=1, f(m \circ n)=f(m) f(n)$ for all coprime $m, n \in H$. Then $\mathcal{M}$ is a group with respect to the Dirichlet convolution $*$ defined as in Section 1 , and for $f \in \mathcal{M}$ the Euler product representation of the Dirichlet series is available,

$$
\widetilde{f}(s)=\sum_{n \in H} \frac{f(n)}{|n|^{s}}=\prod_{p \in P} \widetilde{f}_{p}(s)
$$

where

$$
\widetilde{f}_{p}(s)=1+\frac{f(p)}{|p|^{s}}+\frac{f\left(p^{2}\right)}{|p|^{2 s}}+\ldots,
$$

valid in some half plane $\operatorname{Re} s>\alpha$ of absolute convergence.

For weight functions $\lambda \in W(H, \circ)$ we consider the sets

$$
\mathcal{G}_{\lambda}=\left\{f \in \mathcal{M} \mid \sum_{p \in P} \frac{|f(p)|^{2}}{|p|^{2}} \lambda^{2}(p)<\infty, \sum_{p \in P, \nu \geq 2} \frac{\left|f\left(p^{\nu}\right)\right|}{|p|^{\nu}} \lambda\left(p^{\nu}\right)<\infty\right\},
$$




$$
\begin{aligned}
& \mathcal{G}_{\lambda}^{*}=\left\{f \in \mathcal{G}_{\lambda} \mid \widetilde{f}_{p}(s) \neq 0 \text { for all } p \in P, \text { Re } s \geq 1\right\}, \\
& \mathcal{B}_{\lambda}=\left\{f \in \mathcal{G}_{\lambda} \mid \sum_{p \in P} \frac{|f(p)|}{|p|} \lambda(p)<\infty\right\}, \\
& \mathcal{D}_{\lambda}=\left\{f \in \mathcal{M} \mid \sum_{n \in H} \frac{|f(n)|}{|n|} \lambda(n)<\infty\right\} .
\end{aligned}
$$

In the special case $\lambda=1,(H, \circ)=(\mathbb{N}, \cdot)$ these sets have been introduced and studied by Heppner and Schwarz [8]. For a comprehensive exposition we refer to Schwarz and Spilker [19], Chapter III. In this and the next section their results are considerably extended (see also [11]).

Theorem 2. Let $(H, \circ)$ be an arithmetical semigroup and $\lambda \in W(H, \circ)$. Then:

(i) $\left(\mathcal{G}_{\lambda}, *\right)$ is a semigroup with unit element $\varepsilon$,

(ii) $\mathcal{B}_{\lambda}=\mathcal{D}_{\lambda}$,

(iii) $\left(\mathcal{G}_{\lambda}^{*}, *\right)$ is a group.

Proof. The proof of (i) is an easy exercise in convergence, and the proof of (ii) uses the submultiplicativity (4) of weight functions $\lambda$ in the form

$$
\begin{aligned}
\sum_{n \in H} \frac{|f(n)|}{|n|} \lambda(n) & \leq \prod_{p \in P}\left(1+\sum_{\nu \geq 1} \frac{\left|f\left(p^{\nu}\right)\right|}{|p|^{\nu}} \lambda\left(p^{\nu}\right)\right) \\
& \leq \exp \left(\sum_{p \in P, \nu \geq 1} \frac{\left|f\left(p^{\nu}\right)\right|}{|p|^{\nu}} \lambda\left(p^{\nu}\right)\right) .
\end{aligned}
$$

For the proof of (iii) observe that for $f, g \in \mathcal{G}_{\lambda}^{*}$,

$$
(f * g)_{p}^{\sim}(s)=\widetilde{f}_{p}(s) \widetilde{g}_{p}(s) \neq 0 \quad(p \in P, \operatorname{Re} s \geq 1)
$$

so that $\mathcal{G}_{\lambda}^{*}$ is closed under convolution. Further, if $f, f^{-1} \in \mathcal{G}_{\lambda}$ then also

$$
\widetilde{f}_{p}(s)\left(f_{p}^{-1}\right)^{\sim}(s)=\widetilde{\varepsilon}_{p}(s)=1 \quad(p \in P, \operatorname{Re} s \geq 1)
$$

so that $f, f^{-1} \in \mathcal{G}_{\lambda}^{*}$. Therefore it remains to show that $f \in \mathcal{G}_{\lambda}^{*}$ implies $f^{-1} \in \mathcal{G}_{\lambda}$.

Obviously for $f \in \mathcal{M}$ the Euler product factors $\widetilde{f}_{p}(s)$ of the Dirichlet series $\widetilde{f}(s)$ correspond to the $p$-fibres $f_{p} \in \mathcal{M}$ defined by

$$
f_{p}(n)= \begin{cases}f\left(p^{\nu}\right) & \text { if } n=p^{\nu}, \nu \in \mathbb{N}_{0}, \\ 0 & \text { otherwise, }\end{cases}
$$

and $f \in \mathcal{G}_{\lambda}^{*}$ implies $f_{p} \in \mathcal{G}_{\lambda}^{*}$ for every $p \in P$. Observe that $f_{p} \in \mathcal{G}_{\lambda}^{*}$ yields $f_{p} \in \mathcal{A}_{\omega}^{*}$ where $\omega(\nu)=\lambda\left(p^{\nu}\right)$ since

$$
f_{p}(s)=t_{f_{p}}(z)
$$


for $|z|=|p|^{1-s} \in U$. Hence, by Theorem $1, f_{p}^{-1} \in \mathcal{A}_{\omega}^{*}$, which gives $f_{p}^{-1} \in \mathcal{G}_{\lambda}$ for every $p \in P$. Thereby the proof of (iii) is reduced to carrying over the $\mathcal{G}_{\lambda}$-membership from all $p$-fibres $f_{p}^{-1}$ to $f^{-1}$.

Consider the decomposition of the Euler product

$$
\widetilde{f}(s)=\prod_{|p| \leq c} \widetilde{f}_{p}(s) \cdot \prod_{|p|>c}\left(1-\frac{f(p)}{|p|^{s}}\right)^{-1} \cdot \prod_{|p|>c}\left(1-\frac{f(p)}{|p|^{s}}\right) \tilde{f}_{p}(s)
$$

which corresponds to the decomposition

$$
f=\left(\underset{|p| \leq c}{*} f_{p}\right) * g * h
$$

with $g, h \in \mathcal{M}$ satisfying

$$
\begin{aligned}
& g_{p}(n)= \begin{cases}f^{\nu}(p) & \text { if } n=p^{\nu},|p|>c, \nu \in \mathbb{N}_{0}, \\
0 & \text { otherwise, }\end{cases} \\
& h_{p}(n)= \begin{cases}f\left(p^{\nu}\right)-f\left(p^{\nu-1}\right) f(p) & \text { if } n=p^{\nu},|p|>c, \nu \in \mathbb{N}, \\
1 & \text { if } n=1, \\
0 & \text { otherwise. }\end{cases}
\end{aligned}
$$

For sufficiently large $c>0$ a direct verification gives $g, h \in \mathcal{G}_{\lambda}^{*}$ and

$$
\sum_{p \in P, \nu \geq 2} \frac{\left|h\left(p^{\nu}\right)\right|}{|p|^{\nu}} \leq \frac{1}{2}
$$

Evidently $g^{-1} \in \mathcal{G}_{\lambda}$. From $h^{-1} * h=\varepsilon$ we obtain the recursion formula

$$
h^{-1}\left(p^{\nu}\right)=-\sum_{2 \leq \varrho \leq \nu} h\left(p^{\varrho}\right) h^{-1}\left(p^{\nu-\varrho}\right) \quad(|p|>c, \nu \geq 2)
$$

and $h(p)=h^{-1}(p)=0$ for all $p \in P$, while $h\left(p^{\nu}\right)=0$ for all $p \in P$ with $|p| \leq c$ and $\nu \in \mathbb{N}$. Hence, by considering partial sums,

$$
\begin{aligned}
\Sigma: & =\sum_{p \in P, \nu \geq 2} \frac{\left|h^{-1}\left(p^{\nu}\right)\right|}{|p|^{\nu}} \lambda\left(p^{\nu}\right) \\
& \leq \sum_{p \in P, \nu \geq 2} \sum_{2 \leq \varrho \leq \nu} \frac{\left|h\left(p^{\varrho}\right)\right|}{|p|^{\varrho}} \cdot \frac{\left|h^{-1}\left(p^{\nu-\varrho}\right)\right|}{|p|^{\nu-\varrho}} \lambda\left(p^{\varrho}\right) \lambda\left(p^{\nu-\varrho}\right) \\
& \leq \sum_{p \in P, \nu \geq 2} \frac{\left|h\left(p^{\nu}\right)\right|}{|p|^{\nu}} \lambda\left(p^{\nu}\right)+\sum_{p \in P, \varrho \geq 2, \sigma \geq 2} \frac{\left|h\left(p^{\varrho}\right)\right|}{|p|^{\varrho}} \lambda\left(p^{\varrho}\right) \frac{\left|h^{-1}\left(p^{\sigma}\right)\right|}{|p|^{\sigma}} \lambda\left(p^{\sigma}\right) \\
& \leq(1+\Sigma) \sum_{p \in P, \nu \geq 2} \frac{\left|h\left(p^{\nu}\right)\right|}{|p|^{\nu}} \lambda\left(p^{\nu}\right) \leq \frac{1}{2}(1+\Sigma) .
\end{aligned}
$$

It follows that $\Sigma \leq 1$, and therefore $h^{-1} \in \mathcal{G}_{\lambda}$. By taking the inverse $f^{-1} \in$ $\mathcal{M}$ we see from (6) that

$$
f^{-1}=\left(\underset{|p| \leq c}{*} f_{p}^{-1}\right) * g^{-1} * h^{-1}
$$


is the convolution product of finitely many elements of $\mathcal{G}_{\lambda}$ which, by (i), also belongs to $\mathcal{G}_{\lambda}$.

An immediate consequence of Theorem 2 is the following weighted relationship theorem for multiplicative functions on arithmetical semigroups.

Theorem 3. Let $(H, \circ)$ be an arithmetical semigroup, $\lambda \in W(H, \circ)$. If $g \in \mathcal{G}_{\lambda}$ and $f \in \mathcal{G}_{\lambda}^{*}$ are $\lambda$-related, i.e.

$$
\sum_{p \in P} \frac{|g(p)-f(p)|}{|p|} \lambda(p)<\infty,
$$

then there exists $h \in \mathcal{D}_{\lambda}$ such that $g=h * f$.

Proof. By Theorem 2(i), (iii) we have $h=g * f^{-1} \in \mathcal{G}_{\lambda}$. Since $h(p)=$ $g(p)-f(p)$ for all $p \in P$, the $\lambda$-relationship of $g$ and $f$ implies $h \in \mathcal{B}_{\lambda}$, and by Theorem 2(ii) the proof is complete.

In the special case $(H, \circ)=(\mathbb{N}, \cdot)$ the weighted relationship theorem from [11] holds again. For $\lambda=1$ see also Heppner and Schwarz [8], Schwarz and Spilker [19], Chapter III.

4. A transfer principle. Theorem 3 provides a transfer principle concerning asymptotic formulae and convergent series with multiplicative functions on arithmetical semigroups $(H, \circ)$. The derivation formula for Dirichlet series,

$$
\left(\sum_{n \in H} \frac{f(n)}{|n|^{s}}\right)^{\prime}=-\sum_{n \in H} \frac{f(n)}{|n|^{s}} \log |n|,
$$

valid in any half plane of absolute convergence, suggests considering logarithmic weight functions. For arbitrary arithmetical functions $f: H \rightarrow \mathbb{C}$ we set for abbreviation

$$
N(f, x)=\sum_{n \in H,|n| \leq x} f(n) \quad(x>0) .
$$

Proposition 1. Let $k \in \mathbb{N}_{0}$ and $\lambda(n)=(1+\log |n|)^{k}$ for $n \in H$. Assume that the functions $f \in \mathcal{G}_{\lambda}^{*}$ and $g \in \mathcal{G}_{\lambda}$ are $\lambda$-related. If for certain constants $\alpha, \beta$ with $\operatorname{Re} \alpha \geq \beta \geq 1$ and $l \in \mathbb{N}_{0}$,

$$
N(f, x)=x^{\alpha} P(\log x)+o\left(x^{\beta} \log ^{l} x\right) \quad \text { as } x \rightarrow \infty
$$

with a polynomial $P$ of degree $\leq k$, then also

$$
N(g, x)=x^{\alpha} Q(\log x)+o\left(x^{\beta} \log ^{l} x\right) \quad \text { as } x \rightarrow \infty
$$

with a polynomial $Q$ of degree $\leq k$. In particular, the Dirichlet series $\widetilde{h}(s)$ of $h=g * f^{-1}$ and its derivatives up to order $k$ converge absolutely for $\operatorname{Re} s \geq 1$, and 


$$
Q(t)=\sum_{j=0}^{k} \frac{\widetilde{h}^{(j)}(\alpha)}{j !} P^{(j)}(t)
$$

For the proof notice that $\lambda \in W(H, \circ)$. The absolute convergence of the series

$$
\widetilde{h}^{(j)}(s)=(-1)^{j} \sum_{n \in H} \frac{h(n)}{|n|^{s}} \log ^{j}|n| \quad(\operatorname{Re} s \geq 1)
$$

for $j=0,1, \ldots, k$ is an immediate consequence of Theorem 3. In particular,

$$
\begin{aligned}
(-1)^{j} \sum_{\substack{n \in H \\
|n| \leq x}} \frac{h(n)}{|n|^{\alpha}} \log ^{j}|n| & =\widetilde{h}^{(j)}(\alpha)+O(1) \sum_{\substack{n \in H \\
|n|>x}} \frac{|h(n)|}{|n|^{\operatorname{Re} \alpha}} \log ^{j}|n| \\
& =\widetilde{h}^{(j)}(\alpha)+o\left(x^{1-\operatorname{Re} \alpha} \log ^{j-k} x\right)
\end{aligned}
$$

as $x \rightarrow \infty, j=0,1, \ldots, k$. Now $g=h * f$ implies

$$
\begin{aligned}
N(g, x)= & \sum_{\substack{d \in H \\
|d| \leq x}} h(d) N\left(f, \frac{x}{|d|}\right) \\
= & x^{\alpha} \sum_{\substack{d \in H \\
|d| \leq x}} \frac{h(d)}{|d|^{\alpha}} P\left(\log \frac{x}{|d|}\right) \\
& +x^{\beta} \log ^{l} x \sum_{\substack{d \in H \\
|d| \leq x}} \frac{|h(d)|}{|d|^{\beta}}\left(1-\frac{\log |d|}{\log x}\right)^{l} o(1),
\end{aligned}
$$

where $o(1)$ refers to $x /|d| \rightarrow \infty$. By splitting at $\sqrt{x}$, say, we see that the last sum is $o(1)$ as $x \rightarrow \infty$. Finally, by Taylor's formula,

$$
P\left(\log \frac{x}{|d|}\right)=P(\log x-\log |d|)=\sum_{j=0}^{k} \frac{P^{(j)}(\log x)}{j !}(-1)^{j} \log ^{j}|d|,
$$

and the conclusion follows.

R e mark 1. Proposition 1 remains valid when $o$ in the remainder terms is replaced by $O$.

We mention that Theorem 3 holds for arbitrary norm functions on $H$. If, for example, $n \mapsto|n|$ is replaced by $n \mapsto|n|^{*}=|n|^{\gamma}$ with some constant $\gamma>0$ then $\mathcal{G}_{\lambda}$ is left fixed and

$$
\sum_{n \in H,|n|^{*} \leq x} f(n)=N\left(f, x^{1 / \gamma}\right) .
$$

Hence the formal substitution $x \rightarrow x^{1 / \gamma}$ applies in Proposition 1. 
We shall now consider convergent series indexed by elements of $H$. In the case of conditional convergence it is necessary to agree upon an order of the elements of $H$ which may be obtained by any fixed bijective mapping $\nu \rightarrow n_{\nu}$ from $\mathbb{N}$ onto $H$ which, for example, is (weakly) order-preserving, i.e. $\left|n_{\nu}\right| \leq\left|n_{\nu+1}\right|$ for all $\nu \in \mathbb{N}$.

Proposition 2. Let $k \in \mathbb{N}_{0}$ and $\lambda(n)=(1+\log |n|)^{k}$ for $n \in H$. Assume that the functions $f \in \mathcal{G}_{\lambda}^{*}$ and $g \in \mathcal{G}_{\lambda}$ are $\lambda$-related. If for a certain constant $\alpha$ with $\operatorname{Re} \alpha \geq 1$, the series

$$
\sum_{n \in H} \frac{f(n)}{|n|^{\alpha}} \log ^{j}|n| \quad(j=0,1, \ldots, k)
$$

converge to $S_{j}$ then the series

$$
\sum_{n \in H} \frac{g(n)}{|n|^{\alpha}} \log ^{k}|n|
$$

converges to

$$
\sum_{j=0}^{k}\left(\begin{array}{l}
k \\
j
\end{array}\right)(-1)^{j} \widetilde{h}^{(j)}(\alpha) S_{k-j},
$$

where $\widetilde{h}(s)$ is defined in Proposition 1.

The proof is similar to that of Proposition 1. Here we obtain

$$
\begin{aligned}
\sum_{|n| \leq x} \frac{g(n)}{|n|^{\alpha}} \log ^{k}|n| & =\sum_{j=0}^{k}\left(\begin{array}{c}
k \\
j
\end{array}\right) \sum_{|d| \leq x} \frac{h(d)}{|d|^{\alpha}} \log ^{j}|d| \sum_{|m| \leq x /|d|} \frac{f(m)}{|m|^{\alpha}} \log ^{k-j}|m| \\
& =\sum_{j=0}^{k}\left(\begin{array}{c}
k \\
j
\end{array}\right) \sum_{|d| \leq x} \frac{h(d)}{|d|^{\alpha}} \log ^{j}|d|\left(S_{k-j}+o(1)\right)
\end{aligned}
$$

where $o(1)$ refers to $x /|d| \rightarrow \infty$. Again

$$
\sum_{|m| \leq x} \frac{g(n)}{|n|^{\alpha}} \log ^{k}|n|=\sum_{j=0}^{k}\left(\begin{array}{c}
k \\
j
\end{array}\right) S_{k-j} \sum_{|d| \leq x} \frac{h(d)}{|d|^{\alpha}} \log ^{j}|d|+o(1),
$$

and for $x \rightarrow \infty$ the conclusion follows.

5. Ramanujan series. We shall meet some specific functions on arithmetical semigroups $(H, \circ)$ for which standard notations are partly in use (compare, for example, Knopfmacher [9]). For $\beta \in \mathbb{C}$ the function $I^{\beta}: H \rightarrow$ $\mathbb{C}$ is defined by $I^{\beta}(n)=|n|^{\beta}$, and $1=I^{0}, I=I^{1}$. The Möbius function $\mu$ is the inverse function of 1 , i.e. $\mu * 1=\varepsilon$ and

$$
\mu\left(p^{\nu}\right)=\left\{\begin{array}{ll}
-1 & \text { for } \nu=1 \\
0 & \text { for } \nu>1
\end{array} \quad(p \in P, \nu \in \mathbb{N}) .\right.
$$


The divisor function, the divisor sum function, and the Euler function are given by $d=1 * 1, \sigma=1 * I, \varphi=\mu * I$, respectively. All these functions are multiplicative.

For $a \in H$ we set

$$
\eta_{a}(n)=\left\{\begin{array}{ll}
|n| & \text { if } n \mid a \\
0 & \text { otherwise }
\end{array} \quad(n \in H)\right.
$$

and define the Ramanujan sums $c_{n}(a)$ by

$$
c_{n}(a)=\left(\mu * \eta_{a}\right)(n)=\sum_{d \mid n} \mu\left(\frac{n}{d}\right) \eta_{a}(d) \quad(n \in H) .
$$

Obviously $\eta_{a}$ represents the pointwise product of $I$ with the characteristic function of the set of divisors of $a$. Hence $\eta_{a}(n)$ and $c_{n}(a)$ are both multiplicative functions of $n$, and $c_{n}(e)=\mu(n), c_{n}(n)=\varphi(n)$.

Remark 2. It is well known (Ramanujan [12]) that in the classical case $(H, \circ)=(\mathbb{N}, \cdot)$ the definition $(8)$ is equivalent to that given at the beginning of Section 1:

$$
c_{n}(a)=\sum_{\substack{1 \leq \nu \leq n \\(\nu, n)=1}} e^{2 \pi i \frac{\nu}{n} a} \quad(n, a \in \mathbb{N}) .
$$

For an easy proof denote the right side by $C_{n}(a)$ and observe that

$$
\sum_{1 \leq \nu \leq n} e^{2 \pi i \frac{\nu}{n} a}=\eta_{a}(n)
$$

also can be written in the form

$$
\sum_{d \mid n} \sum_{\substack{1 \leq \nu \leq n \\(\nu, n)=d}} e^{2 \pi i \frac{\nu / d}{n / d} a}=\sum_{d \mid n} \sum_{\substack{1 \leq \varrho \leq n / d \\(\varrho, n / d)=1}} e^{2 \pi i \frac{\varrho}{n / d} a}=\sum_{d \mid n} C_{n / d}(a) .
$$

This shows $\eta_{a}=1 * C$. (a) or equivalently $C$. $(a)=\mu * \eta_{a}=c$. $(a)$, as stated above.

Theorem 4. Let $k \in \mathbb{N}_{0}, \lambda(n)=(1+\log |n|)^{k}$ for $n \in H$, and $\operatorname{Re} \alpha \geq 1$. Assume that $f \in \mathcal{M}$ and $\mu f \in \mathcal{G}_{\lambda}^{*}$. Then the convergence of the series

$$
s_{j}=\sum_{n \in H} \frac{f(n) \mu(n)}{|n|^{\alpha}} \log ^{j}|n| \quad(j=0,1, \ldots, k)
$$

implies the convergence of the Ramanujan series

$$
\sum_{n \in H} \frac{f(n) c_{n}(a)}{|n|^{\alpha}} \log ^{k}|n|=\sum_{j=0}^{k}\left(\begin{array}{c}
k \\
j
\end{array}\right)(-1)^{j} \widetilde{h}^{(j)}(\alpha) s_{k-j}
$$

for every $a \in H$, where $\widetilde{h}(s)$ is the Dirichlet series of

$$
h=(f c .(a)) *(\mu f)^{-1}
$$


and is represented by the finite product

$$
\text { (11) } \begin{aligned}
\widetilde{h}(s)= & \prod_{p^{\nu} \| a}\left(1-\frac{f(p)}{|p|^{s}}\right)^{-1} \\
& \times\left(\left(1-\frac{1}{|p|}\right) \sum_{\varrho=0}^{\nu} \frac{f\left(p^{\varrho}\right)}{|p|^{\varrho(s-1)}}+\frac{1}{|p|}\left(1-\frac{f\left(p^{\nu+1}\right)}{|p|^{(\nu+1)(s-1)}}\right)\right) .
\end{aligned}
$$

Proof. It follows from (7) and (8) that

$$
\begin{aligned}
\sum_{p} \frac{\left|f(p) c_{p}(a)\right|^{2}}{|p|^{2}} \lambda^{2}(p) & =\sum_{p} \frac{|f(p)|^{2}\left|\eta_{a}(p)-1\right|^{2}}{|p|^{2}} \lambda^{2}(p) \\
& =\sum_{p \mid a} \frac{|f(p)|^{2}(|p|-1)^{2}}{|p|^{2}} \lambda^{2}(p)+\sum_{p \nmid a} \frac{|f(p)|^{2}}{|p|^{2}} \lambda^{2}(p)<\infty
\end{aligned}
$$

and

$$
\begin{aligned}
\sum_{p, \nu \geq 2} \frac{\left|f\left(p^{\nu}\right) c_{p^{\nu}}(a)\right|}{|p|^{\nu}} \lambda\left(p^{\nu}\right) & =\sum_{p, \nu \geq 2} \frac{\left|f\left(p^{\nu}\right)\right|\left|\eta_{a}\left(p^{\nu}\right)-\eta_{a}\left(p^{\nu-1}\right)\right|}{|p|^{\nu}} \lambda\left(p^{\nu}\right) \\
& \leq \sum_{\substack{p, \nu \geq 2 \\
p^{\nu-1} \mid a}}\left|f\left(p^{\nu}\right)\right| \lambda\left(p^{\nu}\right)<\infty
\end{aligned}
$$

Hence $f c .(a) \in \mathcal{G}_{\lambda}$ for every $a \in H$. Further $f \mu$ and $f c .(a)$ are $\lambda$-related since

$\sum_{p} \frac{\left|f(p) \mu(p)-f(p) c_{p}(a)\right|}{|p|} \lambda(p)=\sum_{p} \frac{\left|f(p) \eta_{a}(p)\right|}{|p|} \lambda(p)=\sum_{p \mid a}|f(p)| \lambda(p)<\infty$.

So far, the proof does not depend on the weight function $\lambda \in W(H, \circ)$. But the specific choice of $\lambda$ allows us to apply Proposition 2. By replacing $f$ by $f \mu$ and $g$ by $f c$. (a) we obtain (9) and (10). Since $h$ is associated with the Dirichlet series

$$
\widetilde{h}(s)=\frac{(f c .(a))^{\sim}(s)}{(f \mu)^{\sim}(s)}
$$

a short calculation gives the product representation (11)

Remark 3. For every $s \in \mathbb{C}, \operatorname{Re} s \geq 1$, the product $\widetilde{h}(s)$ from (11) represents a multiplicative function of $a \in H$.

Remark 4 . Theorem 4 gives the justification of formally inserting

$$
c_{n}(a)=\sum_{\substack{d, m \in H \\ d \circ m=n}} \eta_{a}(d) \mu(m)
$$

into the left side of (9) and then summing independently over $d \in H, m \in H$. 
COROLlary 1. If, in addition to the assumptions of Theorem 4, the function $f$ is completely multiplicative then

$$
\widetilde{h}^{(j)}(s)=(-1)^{j}\left(1 *\left(f I^{1-s} \log ^{j}|\cdot|\right)\right)(a) \quad\left(a \in H, j \in \mathbb{N}_{0}\right) .
$$

For the proof observe that $(f \mu)^{-1}=f$ in this case. Hence (10) yields $h=f \eta_{a}$, which gives

$$
\widetilde{h}(s)=\sum_{n \in H} \frac{f(n) \eta_{a}(n)}{|n|^{s}}=\sum_{n \mid a} \frac{f(n)}{|n|^{s-1}}=\left(1 *\left(f I^{1-s}\right)\right)(a) .
$$

By taking the $j$ th derivative the assertion follows.

There are many applications of Theorem 4. Assume, for example, the convergence of the series

$$
\begin{gathered}
\sum_{n \in H} \frac{\mu(n)}{|n|}=0, \quad \sum_{n \in H} \frac{\mu(n) \log |n|}{|n|}=-\frac{1}{A}, \\
\sum_{n \in H} \frac{\mu(n) \log ^{2}|n|}{|n|}=-\frac{2 \gamma_{H}}{A^{2}}
\end{gathered}
$$

with the Euler constant $\gamma_{H}$ of $(H, \circ)$. We meet this situation in all arithmetical semigroups $(H, \circ)$ whose elements are nicely distributed with respect to the norm mapping $|\cdot|$ so that the corresponding zeta function

$$
\widetilde{1}(s)=\sum_{n \in H} \frac{1}{|n|^{s}}=\frac{A}{s-1}+\gamma_{H}+\ldots
$$

is holomorphic for $\operatorname{Re} s>1-\vartheta$ with some $\vartheta>0$ except for a simple pole in $s=1$ (compare Knopfmacher [9]). It is well known that the convergence of the series (12) is equivalent to the prime number theorem in $(H, \circ)$ with suitable remainder term estimates. By taking $f=1$ and $\alpha=1$ in Theorem 4 and Corollary 1 we derive from (12) the following formulae.

COROllary 2. Let $(H, \circ)$ be an arithmetical semigroup such that the series (12) converge with the above sums. Then the series

$$
\begin{aligned}
& \sum_{n \in H} \frac{c_{n}(a)}{|n|}=0, \quad \sum_{n \in H} \frac{c_{n}(a)}{|n|} \log |n|=-\frac{1}{A} d(a), \\
& \sum_{n \in H} \frac{c_{n}(a)}{|n|} \log ^{2}|n|=-\frac{1}{A^{2}} d(a)\left(2 \gamma_{H}+A \log |a|\right)
\end{aligned}
$$

converge for all $a \in H$.

Consider the classical case $(H, \circ)=(\mathbb{N}, \cdot)$ where $A=1$ and $\gamma_{H}=\gamma$, the Euler constant. Then the statements of Corollary 2 have been made first by Ramanujan [12]. In particular, formula (2) occurs here again. By taking the 
basic statements concerning the convergence of the series

$$
\sum_{n=1}^{\infty} \frac{\mu(n) \log ^{k} n}{n^{s}} \quad\left(\operatorname{Re} s \geq 1, k \in \mathbb{N}_{0}\right)
$$

from Landau [10] (see $\S 164$ for $\operatorname{Re} s=1$ ) it is quite obvious how to deal with the summation of the series

$$
\sum_{n=1}^{\infty} \frac{c_{n}(a) \log ^{k} n}{n^{\alpha}}
$$

for $\operatorname{Re} \alpha \geq 1, k \in \mathbb{N}_{0}$. The same reasoning can be applied to Dirichlet $L$-series. Specifically, the following series, formed with Dirichlet characters $\chi: \mathbb{N} \rightarrow \mathbb{C}$ modulo $q \in \mathbb{N}$, different from the principal character $\chi_{0}$ modulo $q$, converge (see Landau [10], $\S 102 \mathrm{ff}$.):

$$
L(1, \chi)=\sum_{n=1}^{\infty} \frac{\chi(n)}{n} \neq 0, \quad \sum_{n=1}^{\infty} \frac{\mu(n) \chi(n)}{n}=\frac{1}{L(1, \chi)} .
$$

Since Dirichlet characters $\chi$ are completely multiplicative we may take $f=\chi, \alpha=1, k=0$ in Theorem 4 and Corollary 1 .

Corollary 3. Let $\chi \neq \chi_{0}$ denote a Dirichlet character modulo $q \in \mathbb{N}$. Then the Ramanujan series

$$
\sum_{n=1}^{\infty} \frac{\chi(n) c_{n}(a)}{n}=\frac{1}{L(1, \chi)}(1 * \chi)(a)
$$

converges for every $a \in \mathbb{N}$.

Observe that, for the nonprincipal character $\chi \bmod 4$, we have $L(1, \chi)=$ $\pi / 4$, and $4(1 * \chi)(a)=r(a)$, the number of integer lattice points on the circle of radius $\sqrt{a}$ about the origin (see Hardy and Wright [7], Theorem 278). This gives Ramanujan's formula (3).

6. Ramanujan expansions. In this section we apply Theorem 4 to the problem of expanding given multiplicative functions into pointwise convergent Ramanujan series. For $k \in \mathbb{N}_{0}$ and $n \in H$ set now $\lambda^{k}(n)=(1+\log |n|)^{k}$ and assume that $1 \in \mathcal{G}_{\lambda^{k}}$. With each $g \in \mathcal{G}_{\lambda^{k}}$ having the property that

$$
\left|\widetilde{g}_{p}(1)-\widetilde{1}_{p}(1)\right|<\left|\widetilde{g}_{p}(1)\right| \quad \text { for all } p \in P
$$

associate the function $G \in \mathcal{M}$ defined by

$$
\frac{G\left(p^{\nu}\right)}{|p|^{\nu}}=\frac{\widetilde{1}_{p}(1)}{\widetilde{g}_{p}(1)} \sum_{\varrho \geq \nu} \frac{(\mu * g)\left(p^{\varrho}\right)}{|p|^{\varrho}} \quad(p \in P, \nu \in \mathbb{N}) .
$$

Since $1, \mu, g \in \mathcal{G}_{\lambda^{k}}$ the series $\widetilde{1}_{p}(1), \widetilde{\mu}_{p}(1), \widetilde{g}_{p}(1)$ are absolutely convergent for each $p \in P$, and (13) yields $\widetilde{g}_{p}(1) \neq 0$. Hence the function $G$ is well defined. 
Notice also that $1, g \in \mathcal{G}_{\lambda^{k}}$ and (13) give the existence of a constant $\delta>0$ such that

$$
\delta \leq\left|\frac{\widetilde{1}_{p}(1)}{\widetilde{g}_{p}(1)}\right|<2 \quad \text { for all } p \in P
$$

and that

$$
\lim _{\nu \rightarrow \infty} \frac{G\left(p^{\nu}\right)}{|p|^{\nu}}=0 \quad \text { for all } p \in P .
$$

LEMma 1. Under the above assumptions we have $\mu G \in \mathcal{G}_{\lambda^{k}}^{*}$, and (14) is equivalent to

$$
g\left(p^{\varrho}\right)=\left(\left(G c .\left(p^{\varrho}\right)\right) *(\mu G)^{-1}\right)_{p}^{\sim}(1) \quad\left(p \in P, \varrho \in \mathbb{N}_{0}\right) .
$$

Proof. We have $(\mu G)\left(p^{\nu}\right)=0$ for $p \in P, \nu \geq 2$, and (13), (14) give

$$
\frac{|(\mu G)(p)|}{|p|} \leq 2 \frac{1+|g(p)|}{|p|}+2 \sum_{\nu \geq 2} \frac{\left|g\left(p^{\nu}\right)\right|}{|p|^{\nu}} .
$$

From $1, g \in \mathcal{G}_{\lambda^{k}}$ and by the choice of the weight function $\lambda^{k}$ we see that

$$
\begin{aligned}
\sum_{p \in P} \frac{|(\mu G)(p)|^{2}}{|p|^{2}} \lambda^{2}(p) \ll & \sum_{p \in P} \frac{1+|g(p)|^{2}}{|p|^{2}} \lambda^{2}(p) \\
& +\sum_{p \in P} \frac{|g(p)|}{|p|} \lambda(p) \sum_{\nu \geq 2} \frac{\left|g\left(p^{\nu}\right)\right|}{|p|^{\nu}} \lambda\left(p^{\nu}\right) \\
& +\sum_{p \in P}\left(\sum_{\nu \geq 2} \frac{\left|g\left(p^{\nu}\right)\right|}{|p|^{\nu}} \lambda\left(p^{\nu}\right)\right)^{2} \ll 1 .
\end{aligned}
$$

Further, for $\operatorname{Re} s \geq 1$ and $p \in P$, we see from (13) and (14) that

$$
\begin{aligned}
\left|(\mu G)_{p}^{\sim}(s)\right| & =\left|1-\frac{G(p)}{|p|^{s}}\right|=\left|1-\frac{1}{|p|^{s-1}}\left(1-\frac{\widetilde{1}_{p}(1)}{\widetilde{g}_{p}(1)}\right)\right| \\
& \geq 1-\left|1-\frac{\widetilde{1}_{p}(1)}{\widetilde{g}_{p}(1)}\right|>0 .
\end{aligned}
$$

Hence $\mu G \in \mathcal{G}_{\lambda^{k}}^{*}$.

By explicitly writing down the right side of (15) we obtain

$g\left(p^{\varrho}\right)=\frac{1}{(\mu G)_{p}^{\sim}(1)}\left(\left(1-\frac{1}{|p|}\right)\left(1+G(p)+\ldots+G\left(p^{\varrho}\right)\right)+\frac{1}{|p|}\left(1-G\left(p^{\varrho+1}\right)\right)\right)$.

By subtracting the same representation for $g\left(p^{\varrho-1}\right)$ and dividing by $|p|^{\varrho}$, it follows that

$$
\frac{(\mu * g)\left(p^{\varrho}\right)}{|p|^{\varrho}}=\frac{1}{(\mu G)_{p}^{\sim}(1)}\left(\frac{G\left(p^{\varrho}\right)}{|p|^{\varrho}}-\frac{G\left(p^{\varrho+1}\right)}{|p|^{\varrho+1}}\right) \quad(\varrho \in \mathbb{N}),
$$


and summation over $\varrho \geq \nu$ gives

$$
\frac{G\left(p^{\nu}\right)}{|p|^{\nu}}=(\mu G)_{p}^{\sim}(1) \sum_{\varrho \geq \nu} \frac{(\mu * g)\left(p^{\varrho}\right)}{|p|^{\varrho}} \quad(\nu \in \mathbb{N}) .
$$

In particular, for $\nu=1$,

$$
\frac{G(p)}{|p|}=\left(1-\frac{G(p)}{|p|}\right)\left(\frac{\widetilde{g}_{p}(1)}{\widetilde{1}_{p}(1)}-1\right)
$$

and hence

$$
\frac{\widetilde{1}_{p}(1)}{\widetilde{g}_{p}(1)}=(\mu G)_{p}^{\sim}(1) .
$$

This proves (14). For the converse, observe that the above steps can be reversed.

Remark 5. For $k \in \mathbb{N}$ it is not difficult to show that $G \in \mathcal{G}_{\lambda^{k-1}}$ if $1, g \in \mathcal{G}_{\lambda^{k}}$.

Assume $1 \in \mathcal{G}_{\lambda^{k}}$ for $k \in \mathbb{N}_{0}$, and denote by $\mathcal{R}_{k}$ the class of functions $g \in \mathcal{G}_{\lambda^{k}}$ satisfying (13) and having the property that the series

$$
\sum_{n \in H} \frac{\mu(n) G(n)}{|n|} \log ^{j}|n| \begin{cases}=0 & \text { if } 0 \leq j<k \\ \neq 0 & \text { if } j=k\end{cases}
$$

converge where $G$ is defined in (14).

TheOREM 5. Let $k \in \mathbb{N}_{0}$. Then every $g \in \mathcal{R}_{k}$ possesses a pointwise convergent Ramanujan expansion of the form

$$
g(a)=\sum_{n \in H} \widehat{g}(n) c_{n}(a) \quad(a \in H)
$$

with the coefficients

$$
\widehat{g}(n)=\left(\sum_{m \in H} \frac{\mu(m) G(m)}{|m|} \log ^{k}|m|\right)^{-1} \frac{G(n) \log ^{k}|n|}{|n|} \quad(n \in H)
$$

where $G \in \mathcal{M}$ is given in (14).

Proof. Notice that $\mu G \in \mathcal{G}_{\lambda^{k}}^{*}$ by Lemma 1 . By applying Theorem 4 to $G$ instead of $f$ we obtain

$$
\sum_{n \in H} \frac{G(n) c_{n}(a)}{|n|} \log ^{k}|n|=\sum_{m \in H} \frac{\mu(m) G(m)}{|m|} \log ^{k}|m|\left((G c .(a)) *(G \mu)^{-1}\right)^{\sim}(1) .
$$

It remains therefore to show that for all $a \in H$,

$$
g(a)=\left((G c .(a)) *(G \mu)^{-1}\right)^{\sim}(1) .
$$


Since, by Remark 3, the right side is a multiplicative function of $a \in H$ and, by Lemma 1 , both sides coincide for all prime powers $a=p^{\nu}(p \in P, \nu \in \mathbb{N})$ the proof of Theorem 5 is complete.

In order to decide upon the $\mathcal{R}_{k^{-}}$-membership of functions $g \in \mathcal{G}_{\lambda^{k}}$ the following lemma is often useful.

Lemma 2. Let $1 \in \mathcal{G}_{\lambda^{k}}$, and let $g \in \mathcal{G}_{\lambda^{k}}^{*}$ satisfy (13). Then the functions $\mu G, 1 * g^{-1} \in \mathcal{G}_{\lambda^{k}}^{*}$ are $\lambda^{k}$-related, and $g \in \mathcal{R}_{k}$ if and only if

$$
\sum_{n \in H} \frac{\left(1 * g^{-1}\right)(n)}{|n|} \log ^{j}|n| \begin{cases}=0 & \text { for } 0 \leq j<k, \\ \neq 0 & \text { for } j=k .\end{cases}
$$

Moreover, the values of both series (16) and (18) are equal for $j=k$.

Pr o of. From Lemma 1 and Theorem 2 we have $\mu G, 1 * g^{-1} \in \mathcal{G}_{\lambda^{k}}^{*}$. The $\lambda^{k}$-relationship follows from

$$
\begin{aligned}
\sum_{p \in P} \frac{\left|(\mu G)(p)-\left(1 * g^{-1}\right)(p)\right|}{|p|} \log ^{k}|p| & \\
= & \sum_{p \in P}\left|\frac{g(p)-1}{|p|}-1+\frac{\widetilde{1}_{p}(1)}{\widetilde{g}_{p}(1)}\right| \log ^{k}|p| \\
& \ll \sum_{p \in P}\left(\frac{1+|g(p)|^{2}}{|p|^{2}}+\sum_{\nu \geq 2} \frac{\left|g\left(p^{\nu}\right)\right|}{|p|^{\nu}}\right) \log ^{k}|p| \ll 1 .
\end{aligned}
$$

By taking $\mu G$ and $1 * g^{-1}$ for $f$ and $g$ in Proposition 2 with $\alpha=1$ we obtain the equivalence of (16) and (18) for $0 \leq j \leq k$. Further

$$
\sum_{n \in H} \frac{\left(1 * g^{-1}\right)(n)}{|n|} \log ^{k}|n|=\sum_{n \in H} \frac{(\mu G)(n)}{|n|} \log ^{k}|n|
$$

if the Dirichlet series

$$
\widetilde{h}(s)=\frac{\left(1 * g^{-1}\right)^{\sim}(s)}{(\mu G)^{\sim}(s)}
$$

which is absolutely convergent for $\operatorname{Re} s \geq 1$ assumes the value 1 at $s=1$. This comes as a trivial consequence from (14) since all Euler product factors coincide at $s=1$,

$$
(\mu G)_{p}^{\sim}(1)=\frac{\widetilde{1}_{p}(1)}{\widetilde{g}_{p}(1)}=\left(1 * g^{-1}\right)_{p}^{\sim}(1) \quad(p \in P) .
$$

In order to find for every $k \in \mathbb{N}_{0}$ specific examples $g \in \mathcal{R}_{k}$ we restrict our attention to arithmetical semigroups $(H, \circ)$ such that the corresponding zeta function $\widetilde{1}(s)$ converges absolutely for $\operatorname{Re} s>1,(s-1) \widetilde{1}(s)$ is holomorphic for $\operatorname{Re} s>1-\vartheta$ for some $\vartheta>0$ with

$$
\lim _{s \rightarrow 1}(s-1) \widetilde{1}(s)=A>0,
$$


and

$$
N(\mu, x) \ll x \log ^{-q} x \quad(x \geq e)
$$

for every $q>0$. It is not necessary to study how these assumptions are linked. For the classical case $(H, \circ)=(\mathbb{N}, \cdot)$ we refer to Landau [10], $\S 157$.

Obviously $1 \in \mathcal{R}_{0}$, and $d=1 * 1 \in \mathcal{R}_{1}$ by Lemma 2 and (19). Consider now the generalized divisor function $d_{k} \in \mathcal{M}$ defined by $d_{1}=1, d_{k+1}=d_{k} * 1$ for $k \in \mathbb{N}$.

Proposition 3. Let $k \in \mathbb{N}$. Then, under the above assumptions, we have $d_{k+1} \in \mathcal{R}_{k} \cap \mathcal{G}_{\lambda^{k}}^{*}$, and the coefficients in the Ramanujan expansion (1) of $d_{k+1}$ are given by

$$
\begin{aligned}
& \widehat{d}_{k+1}(n) \\
& =(-1)^{k} \frac{A^{k}}{k !} \cdot \frac{\log ^{k}|n|}{|n|} \prod_{p^{\nu} \| n}\left(\left(1-\frac{1}{|p|}\right)^{k} \sum_{\lambda \geq 0}\left(\begin{array}{c}
k+\nu+\lambda-1 \\
k-1
\end{array}\right) \frac{1}{|p|^{\lambda}}\right)^{-1} .
\end{aligned}
$$

Proof. Notice that for all $k \in \mathbb{N}, j \in \mathbb{N}_{0}$ the estimate

$$
\sum_{\substack{n \in H \\|n| \leq x}} d_{k}^{-1}(n) \log ^{j}|n| \ll_{k, j} x \log ^{-q} x \quad(x \geq e)
$$

holds for every $q>0$. This is (19) for $k=j=1$ and (20) is obtained by induction over $k$ and partial summation. Partial summation also leads to the convergence of

$$
(-1)^{j} \sum_{n \in H} \frac{d_{k}^{-1}(n)}{|n|^{s}} \log ^{j}|n|=\left((s-1)^{k}\left(\frac{1}{(s-1) \widetilde{1}(s)}\right)^{k}\right)^{(j)} \quad\left(k \in \mathbb{N}, j \in \mathbb{N}_{0}\right)
$$

for $\operatorname{Re} s \geq 1$. In particular, for $s=1$ we obtain

$$
\sum_{n \in H} \frac{d_{k}^{-1}(n)}{|n|} \log ^{j}|n|= \begin{cases}0 & \text { if } 0 \leq j<k, \\ (-1)^{k} \frac{k !}{A^{k}} & \text { if } j=k .\end{cases}
$$

Further we have (13) and (18) with $g=d_{k+1} \in \mathcal{R}_{k} \cap \mathcal{G}_{\lambda^{k}}^{*}$ by Lemma 2 since $1 * d_{k+1}^{-1}=d_{k}^{-1}$. From Theorem 5 and (21) we conclude that

$$
\widehat{d}_{k+1}(n)=(-1)^{k} \frac{A^{k}}{k !} \cdot \frac{\log ^{k}|n|}{|n|} D_{k+1}(n),
$$

where

$$
D_{k+1}(n)=\prod_{p^{\nu} \| n}\left(\left(1-\frac{1}{|p|}\right)^{k} \sum_{\lambda \geq 0}\left(\begin{array}{c}
k+\nu+\lambda-1 \\
k-1
\end{array}\right) \frac{1}{|p|^{\lambda}}\right)^{-1}
$$

as stated in Proposition 3.

In particular, for $k=1$ we obtain $D_{2}(n)=1$, and the Ramanujan expansion of the divisor function $d=d_{2}$ given in Corollary 2 results once 
more. For $k=2$ the coefficients in the Ramanujan expansion of $d_{3}$ take the form

$$
\widehat{d}_{3}(n)=\frac{A^{2}}{2} \cdot \frac{\log ^{2}|n|}{|n|} \prod_{p^{\nu} \| n} \frac{|p|}{(\nu+1)|p|-\nu} \quad(n \in H) .
$$

Acknowledgements. The author is grateful to Professor John Knopfmacher for kindly arranging a visiting professorship at the University of the Witwatersrand, Johannesburg, South Africa, from February to April 1993, and for his strong interest in and stimulating conversations about this topic.

\section{References}

[1] R. D. Carmichael, Expansions of arithmetical functions in infinite series, Proc. London Math. Soc. (2) 34 (1932), 1-26.

[2] E. Cohen, Almost even functions of finite abelian groups, Acta Arith. 7 (1962), 311-323.

[3] H. Delange, Sur les fonctions arithmétiques multiplicatives, Ann. Sci. École Norm. Sup. 78 (1961), 273-304.

[4] P. D. T. A. Elliott, A mean value theorem for multiplicative functions, Proc. London Math. Soc. (3) 3 (1975), 418-438.

[5] G. Halász, Über die Mittelwerte multiplikativer zahlentheoretischer Funktionen, Acta Math. Acad. Sci. Hungar. 19 (1968), 365-403.

[6] G. H. Hardy, Note on Ramanujan's trigonometrical function $c_{q}(n)$ and certain series of arithmetical functions, Proc. Cambridge Philos. Soc. 20 (1921), 263-271.

[7] G. H. Hardy and E. M. Wright, An Introduction to the Theory of Numbers, Oxford Univ. Press, London, 1960.

[8] E. Heppner und W. Schwarz, Benachbarte multiplikative Funktionen, in: Studies in Pure Mathematics (To the Memory of Paul Turán), Budapest, 1983, 323-336.

[9] J. Knopfmacher, Abstract Analytic Number Theory, Dover, New York, 1975 (1990).

[10] E. Landau, Handbuch der Lehre von der Verteilung der Primzahlen, 2nd ed. reprint., Chelsea, New York, 1953.

[11] L. Lucht, An application of Banach algebra techniques to multiplicative functions, Math. Z. 214 (1993), 287-295.

[12] S. Ramanujan, On certain trigonometrical sums and their applications in the theory of numbers, Trans. Cambridge Philos. Soc. 22 (1918), 259-276; Collected Papers, Cambridge, 1927, No. 21.

[13] W. Rudin, Real and Complex Analysis, McGraw-Hill, London, 1970.

[14] W. Schwarz, Ramanujan-Entwicklungen stark multiplikativer zahlentheoretischer Funktionen, Acta Arith. 22 (1973), 329-338.

[15] -, Ramanujan-Entwicklung stark multiplikativer Funktionen, J. Reine Angew. Math. 262/263 (1973), 66-73.

[16] —, Über die Ramanujan-Entwicklung multiplikativer Funktionen, Acta Arith. 27 (1975), 269-279.

[17] - Fourier-Ramanujan-Entwicklungen zahlentheoretischer Funktionen und Anwendungen, Festschrift J. W. Goethe-Universität, Frankfurt/Main (1981), 399-415. 
[18] W. Schwarz und J. Spilker, Eine Anwendung des Approximationssatzes von Weierstrass-Stone auf Ramanujan-Summen, Nieuw Arch. Wisk. (3) 19 (1971), 198-209.

[19] - - - Arithmetical Functions, Cambridge University Press, Cambridge, 1994.

[20] F. Tuttas, Über die Entwicklung multiplikativer Funktionen nach RamanujanSummen, Acta Arith. 36 (1980), 257-270.

[21] R. Warlimont, Ramanujan expansions of multiplicative functions, ibid. 42 (1983), 111-120.

[22] A. Wintner, Eratosthenian Averages, Waverly, Baltimore, 1944.

INSTITUT FÜR MATHEMATIK

TECHNISCHE UNIVERSITÄT CLAUSTHAL

ERZSTR. 1

38678 CLAUSTHAL-ZELLERFELD, GERMANY 